Unfallchirurg 2022 $\cdot 125: 95-96$

https://doi.org/10.1007/s00113-021-01131-2

Angenommen: 9. Dezember 2021

(C) The Author(s), under exclusive licence to Springer Medizin Verlag GmbH, ein Teil von Springer Nature 2021

\section{Autor}
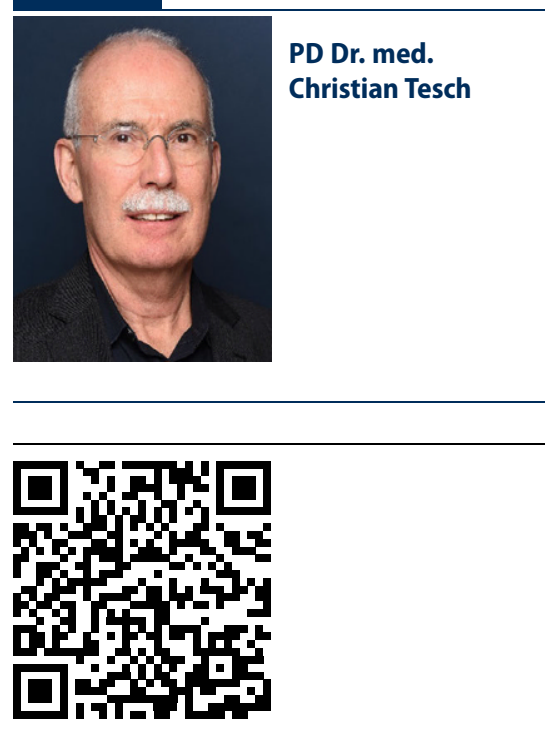

QR-Code scannen \& Beitrag online lesen

\title{
Sonographie in der muskuloskeletalen Chirurgie
}

\author{
Christian Tesch \\ Orthopädie Chirurgie Große Bleichen, Hamburg, Deutschland
}

Ultraschall ist aus der bildgebenden Diagnostik nicht mehr wegzudenken. Sowohl in der inneren Medizin, speziell Kardiologie, als auch in der Gynäkologie ist jeder Facharzt gleichzeitig auch mindestens in der Stufe II der Deutschen Gesellschaft für Ultraschall in der Medizin (DEGUM), also von der wissenschaftlichen Dachorganisation für den Ultraschall, zertifiziert.

Wo steht da die Unfallchirurgie? Die Schockraumsonographie hat sich in Form der e-FAST oder FAST durchgesetzt; sie ist fester Bestandteil des Schockraummanagements in der Unfallchirurgie geworden. Obwohl mit der Sonographie Frakturen durch Darstellung der Unterbrechung des Kortikalisreflexes sicher dargestellt werden können, hat sich diese Methode in der Unfallchirurgie nicht durchgesetzt; nur in der pädiatrischen Traumatologie hat sie nicht zuletzt durch den Einsatz von Ole Ackermann und Kolja Eckert einen Stellenwert erlangt. Die dynamische Untersuchung von Sehnen und Nerven und deren Verletzungen, die zuverlässige Ergussdiagnostik nahezu aller Gelenke und die Stabilitätsdiagnostik mit Ultraschall werden nicht entsprechend ihrer Bedeutung eingesetzt.

Hier wollen wir ansetzen und im aktuellen Themenheft zur Sonographie in der muskuloskeletalen Chirurgie über diese Themen berichten. Wir sind derzeit mit der Erstellung der AWMF-Leitlinie „Fraktur Sonographie" beschäftigt, und Ole Ackermann wird zur Fraktursonographie unter Berücksichtigung der aktuellen Literatur darüber berichten. Hierbei wird besonderer Wert auf die Evidenz der verschiedenen Anwendungsgebiete und deren praktische Durchführung gelegt. Damit wird eine verlässliche „Anleitung“ zur Anwen- dung der Sonographie möglich und damit dazu beigetragen, eine nichtunerhebliche Einsparung von ionisierenden Strahlen zu erreichen.

Interventionelle Techniken zur Punktion von Flüssigkeiten aus Gelenken und periartikulären Räumen gewinnen daneben immer mehr Bedeutung in der praktischen Diagnostik und Therapie unfallchirurgischer und orthopädischer Patienten. Hier werden sowohl die Systematik als auch die Durchführung im Speziellen mit Tipps und Tricks erklärt und dargestellt, was Matthias Wüstner, einem der ersten Chirurgen mit einer umfangreichen Erfahrung und entsprechender Expertise in der interventionellen Sonographie gebührt.

\section{》) Ultraschall ist aus der bildgebenden Diagnostik nicht mehr wegzudenken}

Die Diagnostik bei Pseudarthrosen zur genauen Festlegung des weiteren operativen/nichtoperativen Vorgehens hat sich in den letzten Jahren stark gewandelt, und hierbei ist es besonderes Verdienst des Autors, die Kontrastmittelsonographie ("contrast-enhanced ultrasound", CEUS) zur Diagnostik (Differenzierung infizierter von nichtinfizierter Pseudarthrose) wissenschaftlich so abgeklärt zu haben, dass diese Methode heute als die verlässlichste Diagnostik gelten kann. Julian Doll und Christian Fischer werden zeigen, dass CEUS einfach zu erlernen und sinnvoll in der Klinik einzusetzen ist. Hierbei kommt es nicht darauf an, dass der Unfallchirurg diese Untersuchung persönlich durchführen muss. Wichtig ist, dass er die Technik kennt und es einen qualifizierten Untersucher mit dem dafür 
geeigneten Ultraschallgerät in der Klinik gibt (am einfachsten ein Stufe I der DEGUM zertifizierter Unfallchirurg).

Der "Dauerbrenner" in der unfallchirurgischen Sonographie ist die Abklärung der Verletzungen des Außenbandapparats des oberen Sprunggelenks. Hier wird mit Hartmut Gaulrapp der Protagonist der Untersuchungen des oberen Sprunggelenks im Ultraschall mit aktuellen Hinweisen auf die Evidenz der durchgeführten Untersuchungen berichten.

Das Zertifizierungskonzept der DEGUM in der Sektion Chirurgie ist gut geeignet, Unfallchirurgen so umfangreich weiterzubilden, dass sie die in den nachfolgenden Artikeln berichteten Untersuchungen eigenständig in ihrer Klinik durchführen können. Dieses Konzept wurde bereits 2000 in einer hervorragenden Zusammenarbeit der chirurgischen, internistischen und radiologischen Kollegen entwickelt und dann nachfolgend publiziert [1]. Idealerweise erfolgt dies in der Position eines/ $r$ Oberarztes/Oberärztin mit Schwerpunkt der sonographischen Diagnostik und Intervention. Dies soll keinesfalls bedeuten, dass diese/r Kollegin/Kollege von allen operativen Tätigkeiten entbunden werden sollte, sondern es viel besser im Rahmen seiner/ihrer Kliniktätigkeit als Spezialisierung beherrscht. Damit kann sie/er sowohl eigenständig die weit über das normale Maß hinausgehenden Untersuchungstechniken anwenden als auch die Kolleginnen/Kollegen sachkundig anleiten. Damit wäre der eigentlich für die sonographische Weiterbildung verantwortliche weiterbildungsermächtigte Chefarzt deutlich entlastet und tatkräftig unterstützt. Langfristig wird dadurch auch die Qualität der Sonographie in allen Ebenen der Klinikstruktur verbessert und die ihrer wirklichen Bedeutung angemessene Methode verbreitet und angewendet.

PD Dr. med. Christian Tesch

\section{Korrespondenzadresse}

\section{PD Dr. med. Christian Tesch}

Orthopädie Chirurgie Große Bleichen

Große Bleichen 32, 20354 Hamburg,

Deutschland

tesch@uke.de
Interessenkonflikt. C. Tesch gibt an, dass kein Interessenkonflikt besteht.

\section{Literatur}

1. WorlicekH, StrunkH, Simanowski J(2003)3-dimensional concept for abdominal ultrasonography. Consensus of the section of internal medicine, radiology and surgery of the German society of ultrasonography. Ultraschall Med 24:129-131

\section{Kniearthrose des jungen Patienten}

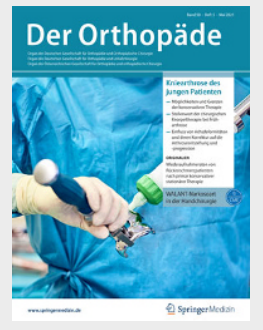

Die Kniearthrose des jungen Patienten stellt noch immer ein großes therapeutisches Problem dar, da Standzeiten und Zufriedenheit vor allem bei jüngeren Patienten deutlich schlechter sind und auch der Wunsch nach sportlicher Belastung nicht immer erfüllt werden kann. Deshalb sollte der Versuch des Gelenkerhaltes an erster Stelle stehen, wobei es wichtig ist, beginnende Arthrose möglichst frühzeitig zu erkennen bzw. Faktoren, die zur Arthrose führen, frühzeitig zu therapieren.

Lesen Sie im Themenheft „Kniearthrose des jungen Patienten“ (Ausgabe 05/2021) von Der Orthopäde mehr zu folgenden Themen:

- Möglichkeiten und Grenzen der konservativen Therapie

- Stellenwert der chirurgischen Knorpeltherapie bei Früharthrose

- Relevanz des Meniskusverlustes für die Arthroseprogression

- Stellenwert der Bandrekonstruktion bei bestehender Arthrose

- Einfluss von Achsdeformitäten und deren Korrektur auf die Arthroseentstehung und -progression

- Fokaler Oberflächenersatz und unikompartimenteller Gelenkersatz

- Bikondylärer Oberflächenersatz des Kniegelenks

Suchen Sie noch mehr zum Thema? Mit e.Med - den maßgeschneiderten Fortbildungsabos von Springer Medizin - haben Sie Zugriff auf alle Inhalte von SpringerMedizin.de. Sie können schnell und komfortabel in den für Sie relevanten Zeitschriften recherchieren und auf alle Inhalte im Volltext zugreifen.

Weitere Infos zu e.Med finden Sie auf springermedizin.de unter "Abos" 